\title{
A Method to Analyze Preferred MTF for Printing Medium Including Paper
}

\author{
Masayuki Ukishima ${ }^{1,3}$, Martti Mäkinen ${ }^{2}$, Toshiya Nakaguchi ${ }^{1}$, \\ Norimichi Tsumura $^{1}$, Jussi Parkkinen ${ }^{3}$, and Yoichi Miyake ${ }^{4}$ \\ 1 Graduate School of Advanced Integration Science, Chiba University, Japan \\ ukishima@graduate.chiba-u.jp \\ 2 Department of Physics and Mathematics, University of Joensuu, Finland \\ 3 Department of Computer Science and Statistics, University of Joensuu, Finland \\ 4 Research Center for Frontier Medical Engineering, Chiba University, Japan
}

\begin{abstract}
A method is proposed to analyze the preferred Modulation Transfer Function (MTF) of printing medium like paper for the image quality of printing. First, the spectral intensity distribution of printed image is simulated by changing the MTF of medium. Next, the simulated image is displayed on a high-precision LCD to reproduce the appearance of printed image. An observer rating evaluation experiment is carried out to the displayed image to discuss what the preferred MTF is. The appearance simulation of printed image was conducted on particular printing conditions: several contents, ink colors, a halftoning method and a print resolution (dpi). The experiments on different printing conditions can be conducted since our simulation method is flexible about changing conditions.
\end{abstract}

Keywords: MTF, printing, LCD, sharpness, granularity.

\section{Introduction}

Image quality of the printed image is mainly related to its tone reproduction, color reproduction, sharpness and granularity. These characteristics are significantly affected by a phenomenon called dot gain which makes the tone appear to be darker. There are two types of dot gain: mechanical dot gain and optical dot gain. Mechanical dot gain is the physical change in dot size as the results of ink amount, strength and tack. Emmel et al. have tried to model mechanical dot gain effect using a combinatorial approach based on Pólya's counting theory 11. Optical dot gain (or the Yule-Nielsen effect) is a phenomenon in printing whereby printed dots are perceived bigger than intended, which is caused by the light scattering phenomenon in the medium layer, where the portion of light transmitted from ink outputs from medium and vice versa as shown in Fig. 1 Optical dot gain causes difficulty to predict the spectral reflectance of print and it produces the reduction in the sharpness of image. It also contributes the reduction in the granularity of image caused by the microscopic distribution of ink dots. The light scattering phenomenon can be quantified by the Modulation

A.-B. Salberg, J.Y. Hardeberg, and R. Jenssen (Eds.): SCIA 2009, LNCS 5575, pp. 607-616, 2009.

(C) Springer-Verlag Berlin Heidelberg 2009 


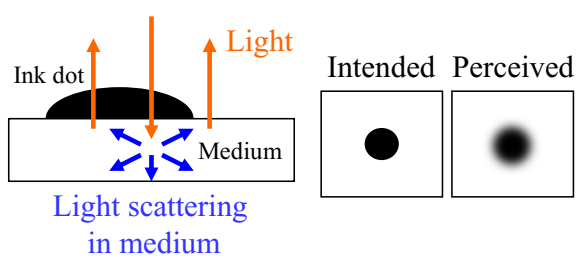

Fig. 1. Optical dot gain

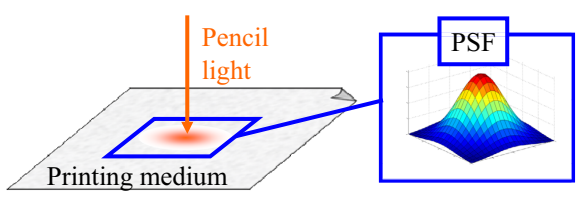

Fig. 2. PSF

Transfer Function (MTF) of medium. The MTF is defined as the absolute value of Fourier transformed Point Spread Function (PSF). The PSF is the impulse response of the system. In this case, the impulse signal is the pencil light like laser and the system is the printing medium as shown in Fig. 2, Because of importance for the image quality control, several researchers have studied the methods to measure and analyze the MTF or PSF of the printing medium [234]. However, discussions have not been done enough about the relationship between the preferred MTF and the printing conditions such as contents, spectral characteristics of inks, halftoning methods, the mechanical dot gain and the printing resolution (dpi). A main objective of this research is constructing a framework of method to simply evaluate the effects of MTF to the printed image. First, we propose a method to simulate the spectral intensity distribution of printed image by changing the MTF of printing medium. Next, we discuss the preferred MTF on particular conditions of printing through the observer rating evaluation experiment which carried out to the simulated print image displayed on a high-precision LCD.

\section{Modulation Transfer Function}

\subsection{MTF of Linear System}

Let a lens system is considered as shown in Fig. 3. For simplicity, we assume that the transmittance of lens is one and the phase transfer of system can be ignored. The output intensity distribution $o(x, y)$ through the lens is given by

$$
\begin{aligned}
o(x, y) & =i(x, y) * \operatorname{PSF}(x, y) \\
& =\mathfrak{F}^{-1}\{I(u, v) \operatorname{MTF}(u, v)\},
\end{aligned}
$$

where $(x, y)$ indicates space coordinates, $(u, v)$ indicates spatial frequency coordinates, $i(x, y)$ is the input intensity distribution whose Fourier transformation is $I(u, v), \operatorname{PSF}(x, y)$ and $\operatorname{MTF}(u, v)$ are the PSF and MTF of the lens system, respectively, * indicates convolution integral operation and $\mathfrak{F}^{-1}$ indicates inverse Fourier transform operation. If the $\operatorname{MTF}(u, v)=1$, the input signal is perfectly transfered through the system: $o(x, y)=i(x, y)$. However, if the value of $\operatorname{MTF}(u, v)$ is decreased as the increase of $(u, v)$, the function $o(x, y)$ becomes to be blurred because of the loss of information at the high spatial frequency area. Therefore, the higher MTF is generally preferred in the linear system, and it is the best case that $\operatorname{MTF}(u, v)=1$. 


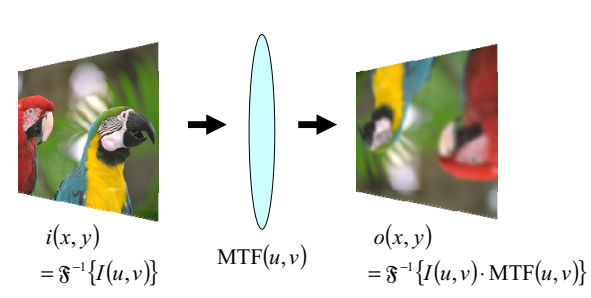

Fig. 3. Lens system

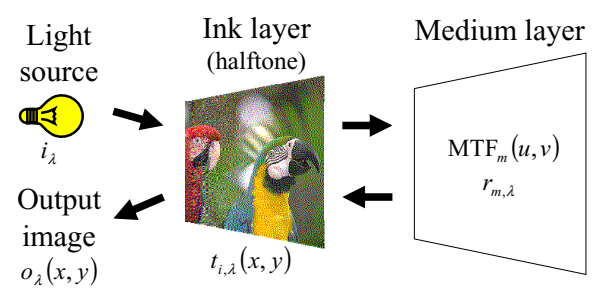

Fig. 4. Printing system

\subsection{MTF of Nonliner System Like Printing Medium}

Let a printing system is considered as shown in Fig. 4 given by

$$
o_{\lambda}(x, y)=i_{\lambda} \mathfrak{F}^{-1}\left\{\mathfrak{F}\left\{t_{i, \lambda}(x, y)\right\} \operatorname{MTF}_{m}(u, v)\right\} r_{m, \lambda} t_{i, \lambda}(x, y),
$$

where the suffix $\lambda$ indicates wavelength, $o_{\lambda}(x, y)$ is the spectral intensity distribution of output light, $i_{\lambda}$ is the spectral intensity of input incidence assumed spatial uniformity, $t_{i, \lambda}(x, y)$ is the spectral transmittance distribution of ink, $\mathrm{MTF}_{m}(u, v)$ is the MTF of printing medium like paper assumed wavelength independency, $r_{m, \lambda}$ is the spectral reflectance of medium assumed spatial uniformity, and $\mathfrak{F}$ indicates Fourier transform operation. Equation (2) is called the reflection image model [7, where the incident light transmits the ink layer, the light is scattered and reflected by the medium layer and transmits the ink layer again. Equation (2) assumes the two layers (ink and medium) are perfectly separable optically, the scattering and reflection phenomena in ink can be ignored, therefore multi reflections between two layers can also be ignored. What is preferred MTF of the medium for image quality in this system? In the case of lens system in previous subsection, the information of image is comprised in the incident distribution $i(x, y)$ and, generally, the information should perfectly be reproduced through the system. On the other hand, in the case of printing system, the information of image is comprised in the ink layer as a halftone image. The half tone image should not be always to reproduce perfectly since it is the microscopic distribution of ink dots causing unpleasant graininess. However, too low MTF may cause the reduction of sharpness of image. Therefore the optimal MTF may exist for the best image quality depending on the printing conditions such as contents, ink colors, halftoning methods and values of the print resolution (dpi). Note that the MTF of medium is different from the MTF of printer. The MTF of printer is the modulation transfer between the input data to the printer and the output response corresponding to $o_{\lambda}(x, y)$. Several methods to measure the MTF of printer has been proposed [5]6.

\section{Apperance Simulation of Printed Image on LCD}

A method is considered in this section to simulate the apperance of the printed image using the 8-bit [0-255] digital color (RGB) image whose resolution is $256 \times$ 256. 


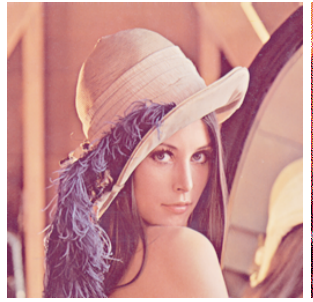

(a) $g_{j}(x, y)$

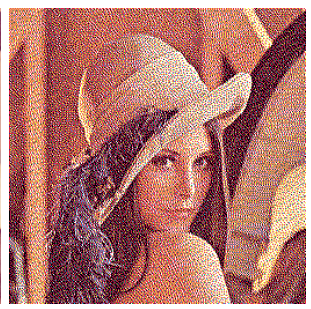

(b) $h_{j}(x, y)$

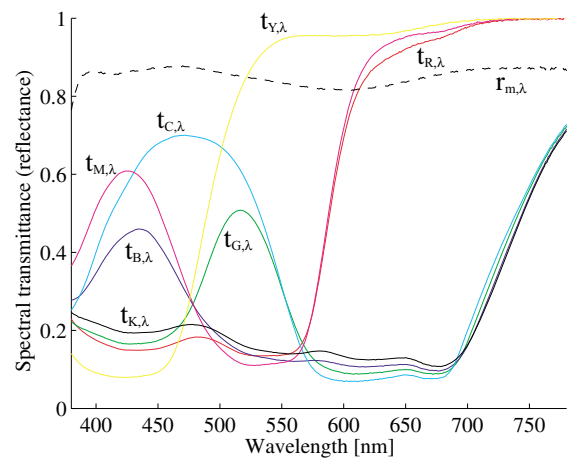

Fig. 5. Digital halftoning

Fig. 6. Spectral transmittance of ink

\subsection{Producing Color Halftone Digital Image}

Assuming that one pixel of the image is printed by four ink dots of $2 \times 2$, the digital image is upsampled from $256 \times 256$ to $512 \times 512$ by the nearest neighbor interpolation [8]. The upsampled image $f_{j}(x, y)$ where $j=R, G$ and $B$ is transformed to the CMY image $g_{k}(x, y)$ where $k=C, M$ and $Y$ :

$$
\begin{aligned}
g_{C}(x, y) & =255-f_{R}(x, y) \\
g_{M}(x, y) & =255-f_{G}(x, y) \\
g_{Y}(x, y) & =255-f_{B}(x, y) .
\end{aligned}
$$

The color digital halftone image $h_{k}(x, y)$ is produced applying the error diffusion method of Floyd and Steinberg [9] to $g_{C}, g_{M}$ and $g_{Y}$, respectively. Figure 5 shows the examples of $g_{j}(x, y)$ and $h_{j}(x, y)$. We used the error diffusion method in this subsection, however, the use of any other halftoning methods do not affect the simulation method described in following subsections. In the real scene of printing, the color change process form RGB to CMY is more complex since it needs the dot gain correction and the gamut mapping from the RGB profile (e.g. sRGB profile) to the print profile. Therefore, the process in this sub-section should be modified as the future work.

\subsection{Estimating Spectral Transmittance of Inks}

Assuming spatial uniformity of ink transmittance for solid prints, the light scattering effect in the printing medium can be ignored mathematically in Eq. (22):

$$
\mathfrak{F}^{-1}\left\{\mathfrak{F}\left\{t_{i, \lambda}\right\} \operatorname{MTF}_{m}(u, v)\right\}=t_{i, \lambda},
$$

and it is derived that

$$
\begin{aligned}
t_{i, \lambda} & =\sqrt{r_{\lambda} / r_{m, \lambda}} \\
r_{\lambda} & =o_{\lambda} / i_{\lambda},
\end{aligned}
$$


where $r_{\lambda}$ is the reflectance of solid print. Therefore, $t_{i, \lambda}$ can be estimated from the measured values of $r_{\lambda}$ and $r_{m, \lambda}$. In this research, seven solid patches were printed on a glossy paper (XP-101, CANON) such as cyan, magenta, yellow, red, green, blue and black using a inkjet printer (W2200, CANON) which is set cyan, magenta and yellow inks (BCI-1302 C, M and Y, CANON). The patches of red, green and blue were printed using two of the three inks, respectively. The patch of black was printed using the three inks simultaneously. The spectral reflectance $r_{\lambda}$ of each solid patch and the spectral reflectance $r_{m, \lambda}$ of the unprinted paper were measured using a spectrophotometer (Lambda 18, Perkin Elmer). Figure 6 shows the estimated $t_{i, \lambda}$ using Eq. (4).

\subsection{Optical Propagation Simulation in Print}

The digital halftone image $h_{j}(x, y)$ produced in Subsection 3.1 can be rewritten to the form $h_{x, y}(C, M, Y)$ having one of the following eight values at each position $[x, y]:(1,0,0),(0,1,0),(0,0,1),(0,1,1),(1,0,1),(1,1,0),(1,1,1)$ and $(0,0,0)$ corresponding to the colors of cyan, magenta, yellow, red, green, blue, black and white (no inks), respectively. By allocating $t_{i, \lambda}$ of each ink estimated in the previous subsection to $h_{x, y}(C, M, Y)$, the spectral transmittance distribution of ink $t_{i,[x, y]}(\lambda)$ can be produced, where $t_{i,[x, y]}(\lambda)$ can be rewritten to the same form in Eq. (2) that is $t_{i, \lambda}(x, y)$. Note that there is no inks at the locations $\left[x_{w}, y_{w}\right]$ where $h_{x_{w}, y_{w}}(C, M, Y)=(0,0,0)$, therefore, $t_{i, \lambda}\left(x_{w}, y_{w}\right)=1$.

Now we have the components $r_{m, \lambda}$ and $t_{i, \lambda}(x, y)$ of Eq. (2). If we define the other components $i_{\lambda}$ and $\mathrm{MTF}_{m}(u, v)$, the output spectral intensity distribution of the print $o_{\lambda}(x, y)$ can be calculated. The incidence $i_{\lambda}$ was assumed to be CIE D65 standard illuminant since we used the LCD whose color temperature is $6500 K$ described in detail in next subsection. We defined the one dimensional MTF of medium given by

$$
\operatorname{MTF}_{m}(u)=\frac{d}{\sqrt{d^{2}+u^{2}}}
$$

where $d$ is a parameter to define the shape of MTF curve. Equation (5) well approximates the MTF of paper as shown in Fig. 7 where this is a example of glossy paper's MTF measured in our previous research [4]. Using Eq. (5), we produced seven types of MTF curve as shown in Fig. 8. Each parameter $d$ is decided in condition that the following formula is equal to $10,25,40,55,70,85$, $100[\%]$, where such parameters $d$ are $0.212,0.756,1.57,2.74,4.62,8.47$ and $\infty$.

$$
\frac{\int_{0}^{10} \mathrm{MTF}_{m}(u) d u}{10} \times 100
$$

Assuming spatial isotropy, two dimensional $\mathrm{MTF}_{m}(u, v)$ was produced using each one dimensional $\mathrm{MTF}_{m}(u)$. Finally, the function $o_{\lambda}(x, y)$ was calculated by Eq. (2) for each $\lambda$. 


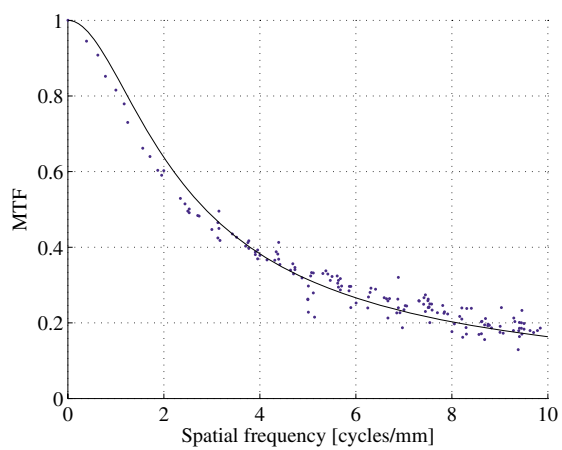

Fig. 7. MTF of a glossy paper

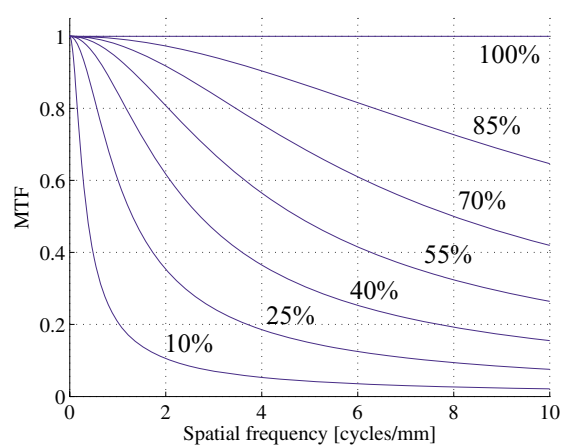

Fig. 8. Generated MTFs

\subsection{Display on LCD and Viewing Distance}

The output intensity distribution of the print $o_{\lambda}(x, y)$ can be rewritten to the form $o_{x, y}(\lambda)$. The spectral function $o_{x, y}(\lambda)$ is converted to CIE RGB tristimulus values given by

$$
\begin{aligned}
R_{x, y} & =\int_{380}^{780} o_{x, y}(\lambda) \bar{r}(\lambda) d \lambda \\
G_{x, y} & =\int_{380}^{780} o_{x, y}(\lambda) \bar{g}(\lambda) d \lambda \\
B_{x, y} & =\int_{380}^{780} o_{x, y}(\lambda) \bar{b}(\lambda) d \lambda,
\end{aligned}
$$

where $\bar{r}(\lambda), \bar{g}(\lambda)$ and $\bar{b}(\lambda)$ are color matching functions [10]. The tristimulus values are displayed on the LCD after the gamma correction given by

$$
V_{x, y}^{\prime}=255 \times\left\{V_{x, y}\right\}^{\frac{1}{\gamma}},
$$

where $V$ is $R, G$ or $B$ and $\gamma$ is the gamma value of LCD. An high-precision LCD (CG-221, EIZO) was used, where the color mode was set to sRGB mode whose gamma value $\gamma=2.2$ and the color temperature is $6500 \mathrm{~K}$. The examples of simulated images are shown in Fig. 9] where the subcaptions (a)-(c) correspond to the applied MTF percentages.

In this simulation, one ink dot is expressed by one pixel of LCD. However, the ink dot size is practically quite smaller than the pixel size. If the printer whose resolution is $600 \mathrm{dpi}$ is assumed, the ink dot size is $4.08 \times 10^{-2}$ [mm/dot]. On the other hand, the pixel size of the LCD is $2.49 \times 10^{-1}$ [mm/pixel]. In order to approximate the appearance of the simulated image to that of the real print, the viewing angles between these were conformed as shown in Fig. 10 by adjusting the viewing distance from the LCD given by

$$
d_{d}=s_{d} d_{p} / s_{p}
$$




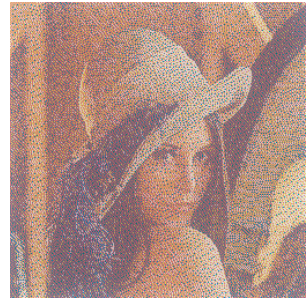

(a) $10 \%$

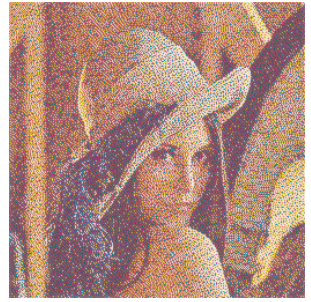

(b) $55 \%$

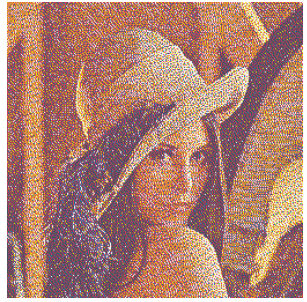

(c) $100 \%$

Fig. 9. Simulated print images

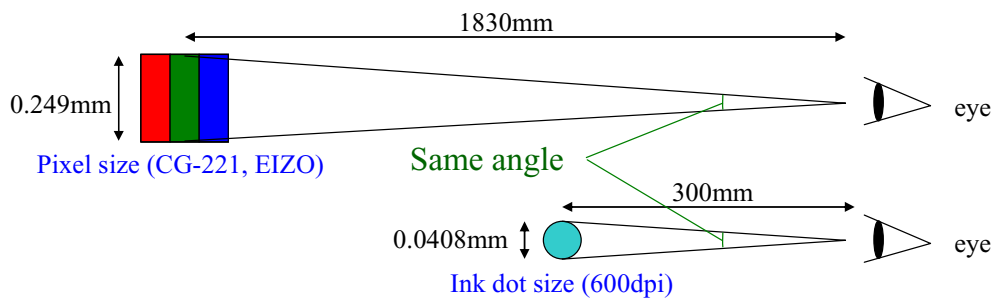

Fig. 10. Viewing distance

where $d_{d}$ and $d_{p}$ are the viewing distance from the LCD and real print, respectively, $s_{d}$ is one pixel size of the LCD and $s_{p}$ is one ink dot size of the real print. Assuming the distance $d_{p}$ is equal to $300[\mathrm{~mm}]$, the distance $d_{d}$ becomes to be equal to about 1830 [mm].

We used not the real print but the LCD for simulation because of several reasons. The objective of this research is to analyze the effects caused by the MTF of medium. However, if we use real medium, other characteristics except the MTF are also changed such as the mechanical dot gain and the color, opacity and granularity of medium. The simulation-based evaluation on display using Eq. (2) can change only the MTF characteristic. The simplicity of observer rating experiment is another advantage using the display. The reason to use the LCD as a display is that the MTF of LCD itself hardly decreases until its Nyquist frequency [1]. Therefore, the MTF of device can be ignored.

\section{Observer Rating Evaluation}

To analyze the preferred MTF of printing medium, an observer rating evaluation test is carried out. Two images simulated in Section 3 are displayed on the LCD simultaneously. We defined seven types of MTF in Subsection 3.3, therefore ${ }_{7} \mathrm{C}_{2}=21$ combinations exist. Subjects evaluate the total image quality of the two images and select the better one. Thurstone's paired comparison method 12 is carried out to the obtained data and the psychological scale are obtained. Three contents were used such as Lenna, Parrots and Pepper [13] as shown in 


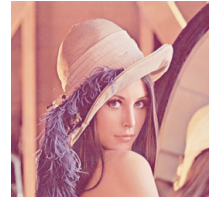

(a) Lenna

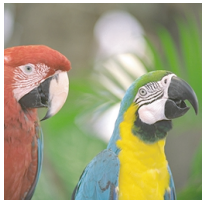

(b) Parrots

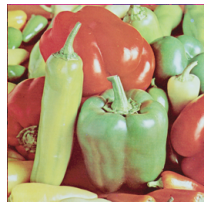

(c) Pepper

Table 1. Paired comparison result (Lenna)

\begin{tabular}{|c||c|c|c|c|c|c|c|}
\hline & $10 \%$ & $25 \%$ & $40 \%$ & $55 \%$ & $70 \%$ & $85 \%$ & $100 \%$ \\
\hline \hline $10 \%$ & 0.50 & 0.85 & 0.80 & 0.65 & 0.70 & 0.50 & 0.40 \\
\hline $25 \%$ & 0.15 & 0.50 & 0.45 & 0.40 & 0.35 & 0.25 & 0.10 \\
\hline $40 \%$ & 0.20 & 0.55 & 0.50 & 0.45 & 0.25 & 0.20 & 0.20 \\
\hline $55 \%$ & 0.35 & 0.60 & 0.55 & 0.50 & 0.20 & 0.15 & 0.00 \\
\hline $70 \%$ & 0.30 & 0.65 & 0.75 & 0.80 & 0.50 & 0.15 & 0.00 \\
\hline $85 \%$ & 0.50 & 0.75 & 0.80 & 0.85 & 0.85 & 0.50 & 0.10 \\
\hline $100 \%$ & 0.60 & 0.90 & 0.80 & 1.00 & 1.00 & 0.90 & 0.50 \\
\hline
\end{tabular}

Fig. 11. Contents

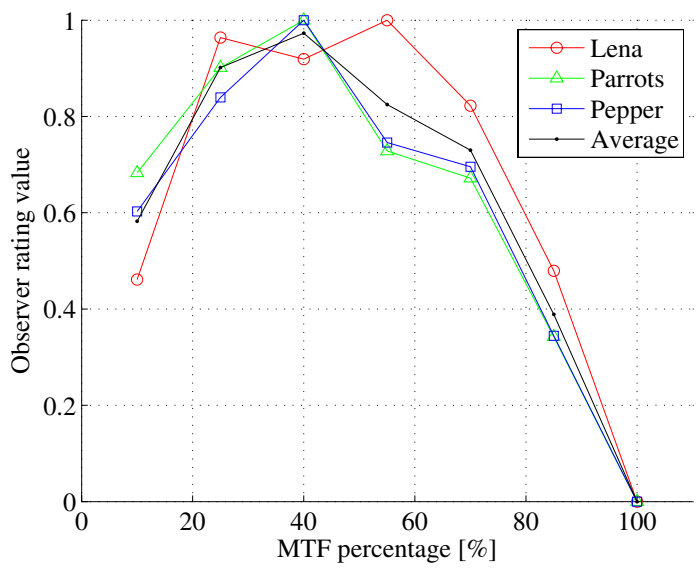

Fig. 12. Observer rating values

Fig. 11. The number of subjects were twenty. The viewing distance was set to $1830[\mathrm{~mm}]$. The evaluation was conducted in a dark room.

Table 1 shows an example of measured result whose content is Lenna, where these percentages are the MTF coverages. For example, the probability, (row, col$\mathrm{umn})=(2,4)=0.40$, indicates that the $40 \%$ of observers evaluated that the MTF coverage of $55 \%$ is better than that of $25 \%$ for the image quality. If the probability is 0.00 or 1.00 , it was converted to 0.01 or 0.99 since Thurstone's method cannot calculate the psychological scale in that case [12. Figure 12 shows the observer rating value of each MTF percentage. The result shows that too low MTF is not preferred and too high MTF is also not preferred. We consider too low MTF causes too low sharpness and too high MTF causes too high granularity caused by the microscopic distribution of ink dots. As the dependence on the contents, the rating results of Parrots and Pepper were similar, however, the rating result of Lenna was different from others. Parrots and Pepper have a commonality about the color 
histogram compared to Lenna. Therefore, it is a possibility that the color histogram affects the preffered MTF of printing medium. The case of using grayscale image should be tested to separate the MTF effects to color and other characteristics such as tone, sharpness and granularity. As the average observer rating value to all contents, it was the best case that the MTF percentage is $40 \%$. However it may significantly depend on the resolution of the print which is $600 \mathrm{dpi}$ in this case. In the case of higher resolution, the granularity of the image is smaller therefore the preferred MTF may become to be higher.

\section{Conclusion}

A method was proposed to simulate the spectral intensity distribution of printed image by changing the MTF of printing medium like paper. The simulated image was displayed on a high-precision LCD to simulate the appearance of image printed on particular conditions: using three contents, dye-based inks, the error diffusion method as the halftoning and a print resolution (600dpi). An observer rating evaluation experiment was carried out to the displayed image to discuss what the preferred MTF is for the image quality of printed image. Thurstone's paired comparison method was adopted as the observer rating evaluation method because of the simplicity of evaluation and high reliability. The main achievement of this research is that a framework was constructed to simply evaluate the effects of MTF to the printed image. Our simulation method is flexible about changing the printing conditions such as contents, ink colors, halftoning methods and the printing resolution (dpi). As future works, we intend to carry out the same kind of experiments on different printing conditions. The case of using grayscale image should be tested to separate the MTF effects to color and other characteristics such as tone, sharpness and granularity. The cases of using other halftoning methods should be tested such as on-demand dither methods and density pattern methods. The simulated printing resolution (dpi) can be changed by changing the viewing distance from the LCD or by using other LCDs having different pixel size (pixel pitch). In this paper, one ink dot of printed image was expressed by one pixel on the LCD. If one ink dot is expressed by multiple pixels on the LCD, the shape of ink dots can be simulated, which can express the mechanical dot gain. We also intend to carry out the physical evaluation using the simulated microscopic spectral intensity distribution $o_{\lambda}(x, y)$.

\section{References}

1. Emmel, P., Herch, R.D.: Modeling Ink Spreading for Color Prediction. J. Imaging Sci. Technol. 46(3), 237-246 (2002)

2. Inoue, S., Tsumura, N., Miyake, Y.: Measuring MTF of Paper by Sinusoidal Test Pattern Projection. J. Imaging Sci. Technol. 41(6), 657-661 (1997)

3. Atanassova, M., Jung, J.: Measurement and Analysis of MTF and its Contribution to Optical Dot Gain in Diffusely Reflective Materials. In: Proc. IS\&T's NIP23: 23rd International Conference on Digital Printing Technologies, Anchorage, pp. 428-433 (2007) 
4. Ukishima, M., Kaneko, H., Nakaguchi, T., Tsumura, N., Kasari, M.H., Parkkinen, J., Miyake, Y.: Optical dot gain simulation of inkjet image using MTF of paper. In: Proc. Pan-Pacific Imaging Conference 2008 (PPIC 2008), Tokyo, pp. 282-285 (2008)

5. Jang, W., Allebach, J.P.: Characterization of printer MTF. In: Cui, L.C., Miyake, Y. (eds.) Image Quality and System Performance III. SPIE Proc., vol. 6059, pp. $1-12(2006)$

6. Lindner, A., Bonnier, N., Leynadier, C., Schmitt, F.: Measurement of Printer MTFs. In: Proc. SPIE, San Jose, California. Image Quality and System Performance VI, vol. $7242(2009)$

7. Inoue, S., Tsumura, N., Miyake, Y.: Analyzing CTF of Print by MTF of Paper. J. Imaging Sci. Technol. 42(6), 572-576 (1998)

8. Gonzalez, R.C., Woods, R.E.: Digital Image Processing, 2nd edn., pp. 64-66. Prentice-Hall, Inc., New Jersey (2002)

9. Ulichhey, R.: Digital Halftoning. MIT Press, Cambridge (1987)

10. Ohta, N., Robertson, A.A.: Colorimetry: Fundamentals And Applications. WileyIs\&t Series in Imaging Science and Technology (2006)

11. Ukishima, M., Nakaguchi, T., Kato, K., Fukuchi, Y., Tsumura, N., Matsumoto, K., Yanagawa, N., Ogura, T., Kikawa, T., Miyake, Y.: Sharpness Comparison Method for Various Medical Imaging Systems. Electronics and Communications in Japan, Part 2 90(11), 65-73 (2007); Translated from Denshi Joho Tsushin Gakkai Ronbunshi J89-A(11), 914-921 (2006)

12. Thurstone, L.L.: The Measurement of Values. Psychol. Rev. 61(1), 47-58 (1954)

13. http://www.ess.ic.kanagawa-it.ac.jp/app_images_j.html 